\title{
Development of a Cloud based Mobile Interactive E-Learning Web Platform for Higher Learning
}

\author{
Georgewill M. Onengiye ${ }^{1}$, Ezeofor J. Chukwunazo ${ }^{2}$ \\ ${ }^{1}$ ken Saro-Wiwa Polytechnic Bori, Rivers State, Nigeria \\ ${ }^{2}$ Department of Electronic \& Computer Engineering, University of Port Harcourt, Rivers State, Nigeria
}

\begin{abstract}
In this paper, development of a cloud based mobile interactive e-learning web platform for tertiary education is presented. The conventional classroom teaching and learning process has posed some challenges to students because of the stress and risk involved to board public bus or car from their various homes and places of residents and converge in the school premises at specified times for lectures. The implication is that these students are always exposed to road hazards everyday transporting themselves from their different locations to school. Schools in rural areas are constrained by materials and teaching aid available, which can negatively affect the learning process. In order to overcome these limitations, Cloud based mobile interactive e-learning system is introduced to address the problems of the conventional teaching and learning process in the tertiary institutions. Now, students can arrange lectures with their instructors/lecturers from distant locations via video conferencing using internet based computers, android phones, tablets etc. and interact more effectively by sharing information and ideas with one another from their respective locations. The e-books needed by students for learning are stored in the cloud server and access given to them to download or read the materials/books from online. The algorithms and block diagrams were designed and the web application developed using php language. The developed web application is tested using lecturers and students' web service platforms which enhanced the learning process and induced safety to the lives of our students.
\end{abstract}

Keywords: Mobile learning, cloud based, PHP-Language, tertiary education, web services

\section{Introduction}

Online learning is a subject related to the virtualized distance learning by means of electronic communication mechanisms, especially over the internet. It is based on the use of approaches with diverse functionalities such as emails, web pages, forums, conferences and so on, to support the process of teaching and learning across the globe. The cloud computing environment rises as a natural platform to provide support to the distance learning systems. Cloud computing is one of the most significant achieved development in the IT industry after the introduction of the personal computer and the internet [1]. The advent of cloud computing is a landmark in the IT industry and has raised keen interest to industrial community and academia. It is a new virtualization model for quick provisioning of shared resources, softwaree $s$, applications and services over the internet to satisfy the elastic demand of the customer with minimum interaction or supervision by the service provider. Thus, cloud computing has become an interesting and increasingly essential trend.

The conventional classroom teaching and learning process which has been in vogue for a long period of time has been seen as the most functional instructional medium. This is because it is a method that most people had grown up to witness and also it has a nature of direct interaction between the teacher and students. For this direct interaction to be achieved with the conventional teaching method, it means that both the teacher and students must convene in a building, usually in the classroom, for the learning process to take place. This poses some challenges due to the fact that all the students would always have to leave their various homes and places of operation and converge in the school premises at specified times for lectures to take place. The implication of this is that these students are always exposed to road hazards and spend valuable time everyday transporting themselves from their different locations to their schools. In areas where there are no good roads, means of transportation becomes a major educational setback.

Another limitation of the classroom method is access to teaching and learning materials. In some schools, particularly in rural areas, which are constrained to the amount of instructional materials and teaching aids, these can negatively impact in the teaching process and learning outcomes. Student would not have good reading materials at their disposal and also those engaged in some form of research work will not have access to current and trending materials which will limits the content and scope of their research [2]. With the advent of the internet and computer technologies, elearning systems have been put in place to address the limitations of the conventional teaching and learning process. No longer would direct face to face interaction be necessary for teaching and learning to take place. Students can collaborate with one another and with their instructors from their various distant locations using their computers having internet connection and interact more effectively and share information and ideas with one another. The e-learning systems however, still have some inherent problems which include restricted mobility features (as with the desktop PCs), lack of scalability to meet future computing needs, etc. We are presently in a mobile communication era where miniaturization is the order of the day. Computers and computing devices are made more portable and can be carried about more easily. With this development, we can take great advantage to apply mobile computing in the learning process which will better enhance the learning process and outcome. 


\section{International Journal of Science and Research (IJSR) \\ ISSN (Online): 2319-7064}

Index Copernicus Value (2013): 6.14 | Impact Factor (2015): 6.391

\section{Related Work}

\subsection{Story Telling (Oral Tradition)}

This is the first recorded method that was adopted as an instructional method for teaching and learning. "Long before the Europeans arrived, formal education had not been part of education. The children were taught about their culture, social activities, survival skills and work. Most of these education processes were imparted to the children informally"[3]. Prior to the invention of reading and writing, people lived in an environment in which they struggled to survive against natural forces, animals and other humans. To survive, preliterate people developed skills that grew into cultural and educational patterns. For a particular group es culture to continue into the future, people had to transmit it, or pass it on, from adults to children. The earliest educational processes involved sharing information about gathering food and providing shelter; making weapons and other tools; learning language; and acquiring the values, behavior, and religious rites or practices of a given culture.

\subsection{Conventional or Classroom}

The traditional or classroom method of teaching and learning, just like the story telling method, involves oral discussions and the physical presence of the teacher and his students for the learning process to occur. However, unlike the first method, the participants are situated in a building (usually a classroom, laboratory, workshop, etc) and other instructional tools which include: textbooks, drawings for illustration, writing materials, most importantly the black board are used in the teaching and learning process. "The first blackboard was invented by James Pillans in the 1800s. He worked as the headmaster of the Old High School of Edinburgh, Scotland. The students in his school used slates with wooden frames and wrote with another slate on it. This slate had wooden frames to protect them from breaking. He took slates and hung them on the wall to teach them Geography and this brought him an idea of the blackboard. The invention of the blackboard had an enormous impact on classroom efficiency. Due to their simplicity, effectiveness, economy and ease of use, the simple blackboard has substantial advantages over any number of more-complex modern technologies [4].

\subsection{The Electronic (or E-Learning)}

The term e-learning has only been in existence since 1999, when the word was first utilized at a CBT systems seminar. Other words also began to spring up in search of an accurate description such as "online learning" and "virtual learning". However, the principles behind e-learning have been well documented throughout history, and there is even evidence which suggests that early forms of e-learning existed as far back as the $19^{\text {th }}$ century" [5]. With the introduction of the computer and internet in the late $20^{\text {th }}$ century, e-learning tools and delivery methods expanded. The first MAC in the $1980^{\circ e} \mathrm{~s}$ enabled individuals to have computers in their homes, making it easier for them to learn about particular subjects and develop certain skill sets. Then, in the following decade, virtual learning environments began to truly thrive, with people gaining access to a wealth of online information and e-learning opportunities. Unlike the traditional or classroom method where participants are all housed in the same building, the e-learning method does not necessarily require a face to face interaction. The physical presence of the tutor and his student may not be relevant. With the use of the PCs, either desktops or laptops having network connections, depending if it is a LAN (Local Area Network) connection or WAN (Wide Area Network), their distance of separation could range from different rooms in the same building to as far as countries apart. This is where the name, distance learninge has its roots.

\subsection{The Mobile (or M-Learning)}

Research is always work in progress. After the successful adoption and implementation of the e-learning platform where students using their computers can enroll for and partake in online courses, work is still ongoing to improve upon this. In the m-learning method, which by application and functionality is a subset of e-learning, activities are carried out using mobile computing devices such as Netbooks, PDAs (Personal Digital Assistants), Mobile phones, Tablet PCs, etc. These devices are miniaturized and are connected over a wireless network which gives rise to portability and mobility features. M-learning is characterized by the use of wireless communication technology, mobile terminals, data processing and storage devices to enable interactive teaching and learning activities to be carried out conveniently, flexibly and efficiently. With the advent of Information and Communication Technology (ICT), mobile learning is rapidly gaining grounds as a very important tool in the educational environment [6-7].

\section{Method}

The design method deployed is top-down approach, where the general functionality of the entire system is described starting from the complex to the basic simpler constituents that make up the system. The design of the mobile learning platform for the lecturer and student interaction comprises two basic components namely software and hardware components.

\subsection{Software Components}

The software components constitute the application program design and the codes used for the actualization of the mlearning platform. The application environment is developed using a set of programming languages and software s namely PHP (PHP Hypertext Preprocessor), MySQL (Structured Query Language), HTML (Hyper Text Markup Language) and the WAMP server software.

A. PHP: PHP (PHP Hypertext Preprocessor) is the programming language used for the application development. It is a server-side scripting language embedded in HTML. It is basically used to connect from the website to the database. The choice of the PHP Language is for the following reasons: 1)It runs on a wide range of platforms and operating systems such as Android, Windows, Linux and UNIX.

\section{Volume 5 Issue 7, June 2016} www.ijsr.net 


\section{International Journal of Science and Research (IJSR) \\ ISSN (Online): 2319-7064}

Index Copernicus Value (2013): 6.14 | Impact Factor (2015): 6.391

2)It is compatible with almost all servers in use today: Apache, IIS, etc.

B. MySQL: MySQL is a Relational Database Management System (RDMS) that relies on SQL for processing the data in the database. It is a fast and user friendly application. MySQL provides APIs for the Java and PHP languages (including $\mathrm{C}$ and $\mathrm{C}++$ ). MySQL is most commonly used for Web applications and for embedded applications and has become a popular alternative to proprietary database systems because of its speed and reliability. MySQL can run on UNIX, Linux, Windows and Mac OS.

C.HTML: HTML (Hyper-Text Markup Language) is used for the web design. HTML is based on HTTP (Hyper-Text Transfer Protocol). This protocol is used to link the various web pages on the designed website using hyperlink.

D.WAMP Server Software: WAMP (Windows, Apache, MySQL, and PHP) is the Windows operating system based program that installs and configures Apache web server, MySQL database server and PHP scripting language. It offers an easy way to install Apache, PHP and MySQL packages with an easy to use installation program. WAMP is so user-friendly because once it is installed it is ready to go. No need to do any additional configuring or modification of any configuration files to get it running.

\subsection{Hardware Components}

The hardware part comprises all the physical devices and also the network elements that are used in the mobile learning process. These include: Handheld devices ranging from iPads, mobile phones, Tablet PCs, Personal Digital Assistants (PDAs), net books, etc that are used in the interactive learning session. These mobile devices, however, are not designed but are devices to be utilized in demonstrating the cloud-based m-learning. The network infrastructure is also a vital aspect of the hardware components since it comprises the telecommunication network, the internet, wireless networks, cloud service infrastructure constituted of servers, gateways, etc through which data services are being transported.

\subsection{System Software Design}

The software design for the system uses the block diagram (figure 1) in which the various components that constitute the m-learning platform which include the login or authentication module, the admin or security module, the lecturers "e module and the student module are represented.

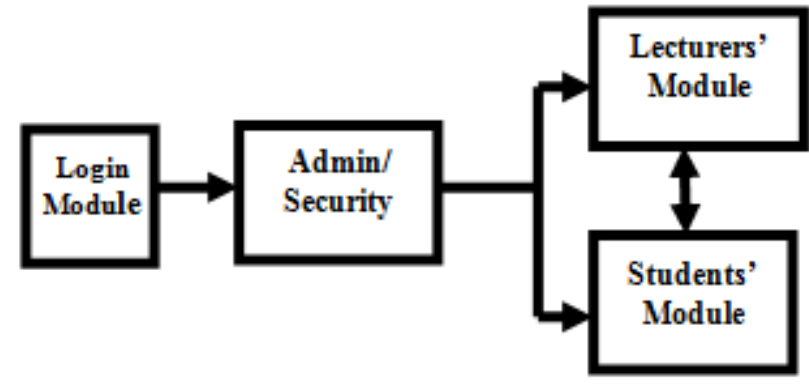

Figure 1: Software design approach

\subsubsection{System Login Module}

The login module is the user authentication module whose primary function is for identity validation. This module is usually the first interface for anyone trying to gain access or make use of the m-learning platform. To login, a username and password is required. These login IDs are usually provided by the admin during the registration process which is stored in a database. The user is required to supply the correct user IDs to gain access. A comparison is done with the information in the data base and what the user supplies, if they match, then the user is granted access, else he is prompted to supply again the correct login IDs.

A. Login Algorithm: The algorithm of a program refers to its logic. It is the step by step description how to arrive at the solution to a problem. It defines a sequence of instructions that when executed in the specified sequence, the desired results are obtained. The algorithm below defines the steps for the login process:

Step 1: START

Step 2: Launch the M-learning platform

Step 3: Enter username and password

Step 4: Validate login details

IF username and password are correct,

THEN go to STEP 5

ELSE go back to STEP 3

Step 5: Launch the M-learning platform

Step 6: STOP.

B.Login Flow Chart: The flow chart for the login process is shown (figure 2)

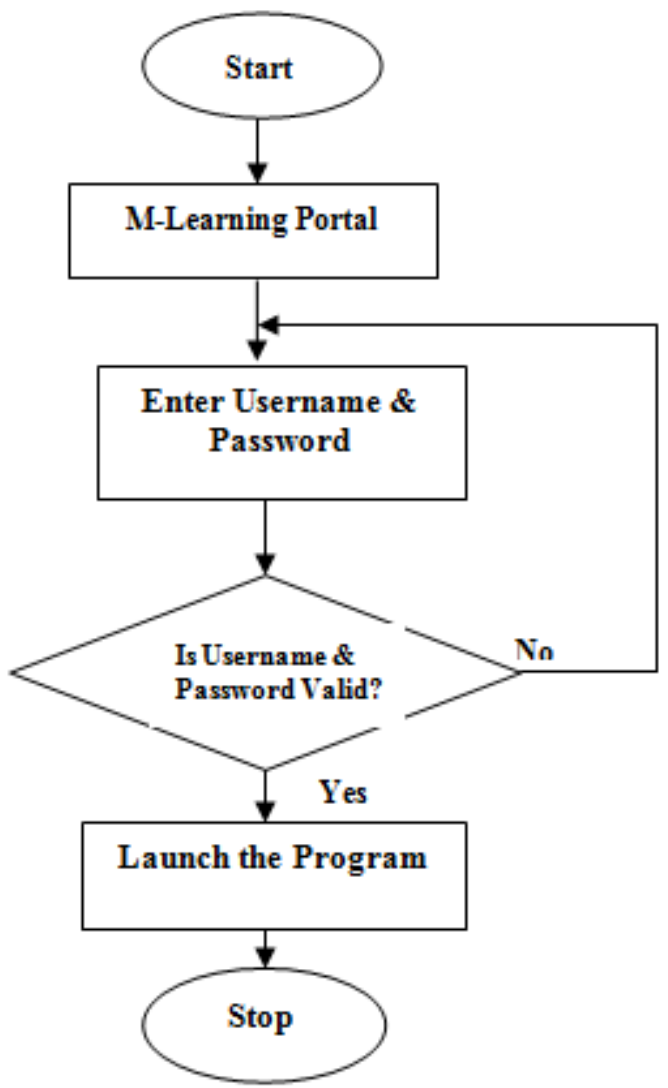

Figure 2: System Login flow chart

Volume 5 Issue 7, June 2016 www.ijsr.net 


\section{International Journal of Science and Research (IJSR) \\ ISSN (Online): 2319-7064}

Index Copernicus Value (2013): 6.14 | Impact Factor (2015): 6.391

\subsubsection{Admin Module}

The Admin module is designed to manage the other modules in the m-learning platform which include the lecturers ${ }^{\text {ee }}$ module and the students ${ }^{\text {ee }}$ module. It is central to the two modules. The admin module is where user registration is done for both the students and the lecturers. For the lecturers, usernames and passwords are being created and supplied as their login details. The student registration, registration numbers are supplied to the students and are required during the login process.

A. Admin Algorithm: The algorithm used to realize the administrator interface is shown below:

Step 1: START

Step 2: Login as Admin

Step 3: Validate Admin IDs

IF username and Password are correct

THEN Go To STEP 4

ELSE Go back To STEP 2

Step 4: View Admin Page

Step 5: Create User Accounts

Step 6: Confirm all fields are completed

IF all fields are completed;

THEN: Go To STEP 7

ELSE: Go back To STEP 5

Step 7: STOP

B.Admin Flow Chart: (Figure 3) shows the admin flow chart.

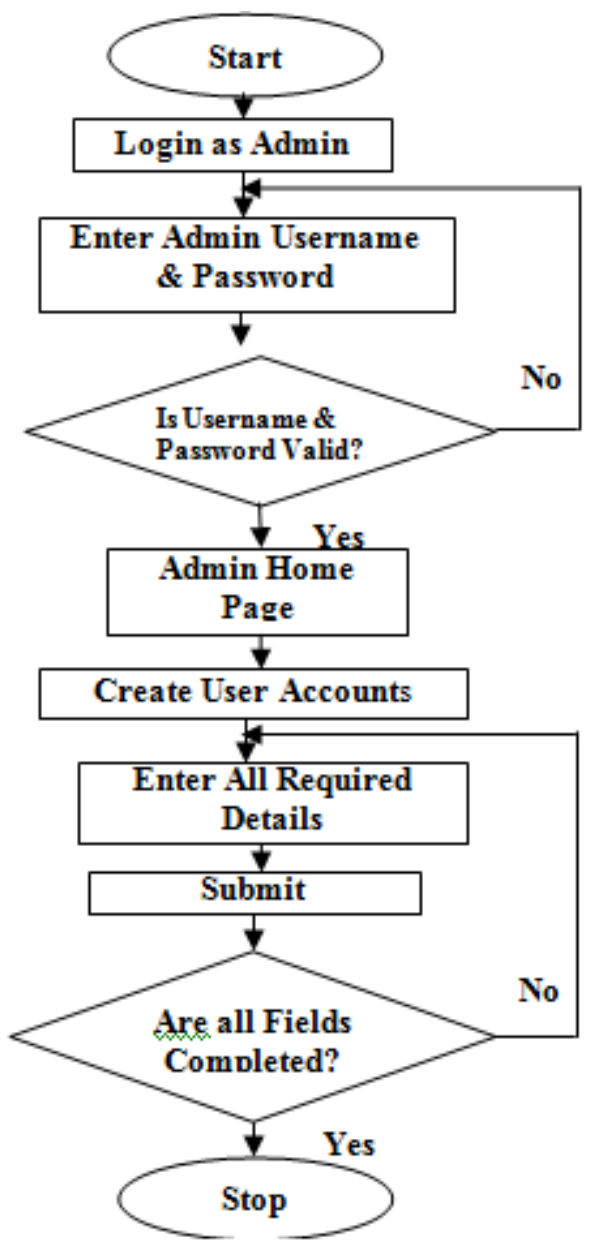

Figure 3: Admin flow chart

\subsubsection{Lecture Module}

This is a very key module in the mobile learning platform design. It is an environment where courses are being taught by the lecturer. A lecturer logs in with his registered username and password and is granted access to this portal. All required courses can be taught on this platform for registered students by using forum chat. There is also provision for the lecturer to upload lecture notes, tests and assessment questions as attachments for reference by the students.

A.Lecture Algorithm: The algorithm for lecture is as follows:

Step 1: START

Step 2: Input lecturers username and password

Step 3: Validate user IDs

IF username and Password are correct

THEN Go To STEP 4

ELSE Go back To STEP 2

Step 4: View Lecturer homepage

Step 5: Lecture session

Step 6: IF lecture time has elapsed

THEN: Go To STEP 7

ELSE: Continue with STEP 5

Step 7: Question and Answer session Step 8: STOP

B.Lecture Flow Chart: (Figure 4) shows the lecture flow chart

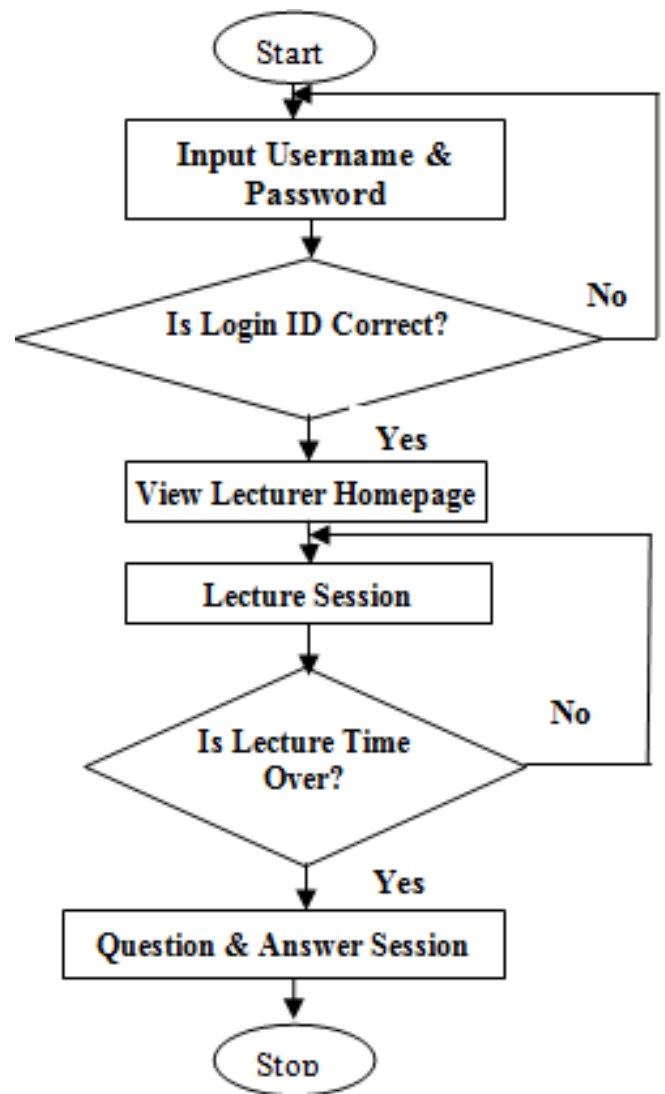

Figure 4: Lecture Flow Chart

\subsubsection{Students Module}

The student portal is a platform for a user logged in as a student to have the privilege to participate in online lectures. To login to the portal, the registered student is prompted for

Volume 5 Issue 7, June 2016 www.ijsr.net 


\section{International Journal of Science and Research (IJSR) \\ ISSN (Online): 2319-7064}

Index Copernicus Value (2013): 6.14 | Impact Factor (2015): 6.391

his username and password. When the login IDs is supplied, they are crosschecked with what is stored in the database. If it matches the input, the user is granted access as a registered student. On the student portal, the lecture notes, assessments, etc. posted by the lecturer can be downloaded and viewed or saved on the students ${ }^{\text {ee }}$ device for further reading and analysis. There is also a link to submit assessments and exams on the platform.

A.Student Algorithm: The algorithm for student is as follows:

Step 1: Start

Step 2: Launch the Student portal

Step 3: ENTER username and password

Step 4: Validate Login IDs

IF username and Password are correct

THEN Go To STEP 5

ELSE Go back To STEP 3

Step 5: View Student Home Page

Step 6: STOP

B.Student Flow Chart: (Figure 5) shows the student flow chart

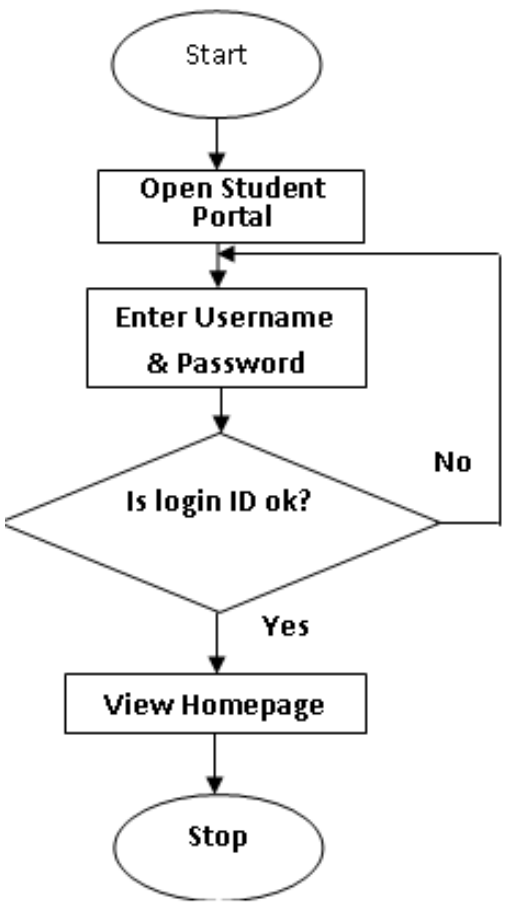

Figure 5: Student Flow Chart

When a student opens the student portal he is prompted to login. If this student is a registered user, then he simply supplies his username and password otherwise he has to sign up or create a login account by filling and submitting the necessary student details. If a student forgets his password and needs to recover it, there is provision on the portal for password recovery. The student is prompted for his registration number and phone number details. In addition to this, a captcha security system is included. The captcha system is alphanumeric and case-sensitive which requires human reasoning to figure out. This system is added to verify that the user is human and not a robot. When these three levels of authentication are passed, the user"s password can be regenerated.

\subsection{Cloud Based M-Learning Architecture \& Operations}

This architecture is the typical implementation scenario of M-learning for higher education in a cloud computing environment. The main objective of Mobile-Learning in the cloud environment is to provide learners the knowledge from the centralized shared resources at anytime and anywhere. The Mobile Distance Learning architecture (figure 6) incorporates communication between end-user devices (terminals) and the Data Center in a cloud computing environment. The terminals can be connected to the Infrastructure inside the University Local Area Network (LAN) or preferably can be connected on external networks (the internet cloud). The University Platform Server hosts educational resources and it is connected on the University LAN. A user may access the platform directly from the University LAN or through the Internet in order to collect the learning materials. The user can access the Data center either from University LAN, or directly from the internet. The authentication server will manage the authorized access to the Data Center, and it will be directly connected on both passive and active servers.

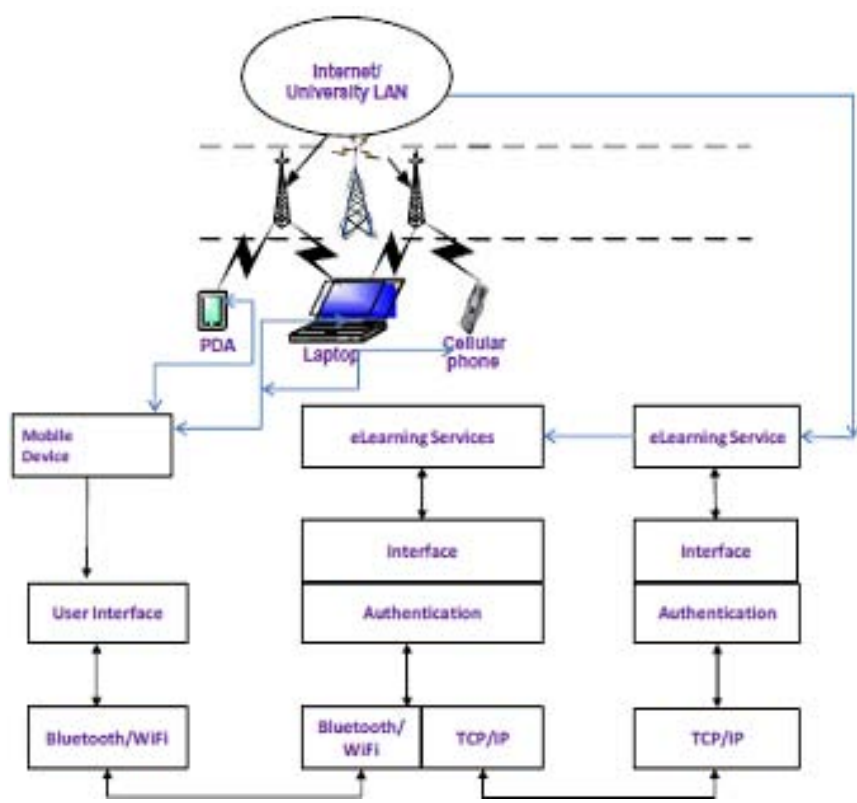

Figure 6: Cloud Based M-Learning Architecture

The server takes additional daa from the storage area network that needs to be processed. The advantage of this architecture is that it offers an interactive mode on mobile devices, as a special benefit from using the Data center within the mobile cloud environment. Another advantage of this model is that it can provide service continuity, or seamless mobility as the user handovers from the external network to the University Local area network. According to this architecture the University classrooms will be connected to the Server Platform and the internet. The University Classroom usually should have the following equipment: A PC, or laptop, microphone, speakers, tablet, webcam, projector, and a monitor, or screen. At the University Classroom the lecturer will present and deliver the content of the learning material to the students in a classical manner, or via the internet to the students that are at home, at work, or simply they are mobile. The students that are at home, or at work connect to the 


\section{International Journal of Science and Research (IJSR) \\ ISSN (Online): 2319-7064 \\ Index Copernicus Value (2013): 6.14 | Impact Factor (2015): 6.391}

course by using their PCs, or laptops using the high speed internet from their homes, or their offices. On the other hand, the mobile students use their mobile devices (mobile smart phones, or tablets) to connect to the course via their mobile networks such as GPRS, UMTS, HSPA, WiFi, WiMAX or LTE.

\subsection{Database \& Hosting of the M-Learning Application Online}

The database functions at the physical layer of the model. It is the storage location of all data content and information. The database server is where the details of each registered student, all lecture materials and notes are stored and retrieved on demand. For the purpose of this design, the database is hosted on the Microsoft apache server which is a reliable database server for cloud applications. The application of the cloud broadens the versatility, scalability and flexibility of the web application. In traditional elearning systems, the website could be hosted on a singular and localized server. This limits the speed and capabilities of all hosts to the processing power of the single server. However, with cloud computing, the database is hosted on cloud resources provided by a Cloud Service Provider (CSP). In this architecture, basic information and applications are stored and retrieved from distributed servers. The implication of this is that larger storage space, faster processing speeds and higher computational power and zero down time is guaranteed.

The M-learning software is an internet based platform and as such is hosted on an online server to be readily accessible by the intended users. Unlike the traditional client-server model where the application software and database is hosted on a single and localized server, this application is hosted on a cloud server. The cloud platform is hosted on the Microsoft Apache Server. The cloud server provides a distributed storage and retrieval system across multiple servers.

\section{Test and Result}

The development of the mobile learning platform makes it clear that the M-learning process enhances learning outcomes of students significantly. A greater advantage is the integration of cloud computing into the learning infrastructure. With cloud computing, not only would physical presence of the teachers and students matter less, but also information storage and retrieval is made very dynamic due to the distributed storage features of the cloud resources. No longer would storage be localized to a particular server or information processed by a singular computer. With cloud computing a vast array of computers and integrated servers can synergize to handle a particular computational task on demand and also guarantee large storage capabilities.

\subsection{The Login Screen Portal}

The login screen is the first platform on the portal. It is an authentication environment (figure 7) where users are prompted to supply their relevant IDs for validation. The login screen is very essential because it performs security functions such that only registered persons can be allowed access to their user accounts.

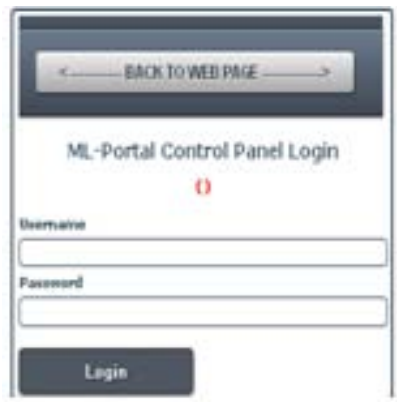

Figure 7: Login Portal

\subsection{Admin Home Page Portal}

The home page is usually the first environment to display after logging in. To open the home page, enter the URL: $\mathrm{http} / / /$ www.uniportmlearningportal.com/apps in the address bar. A login authentication window is prompted. The admin then inputs his username and password and is granted access to the home screen (figure 8).

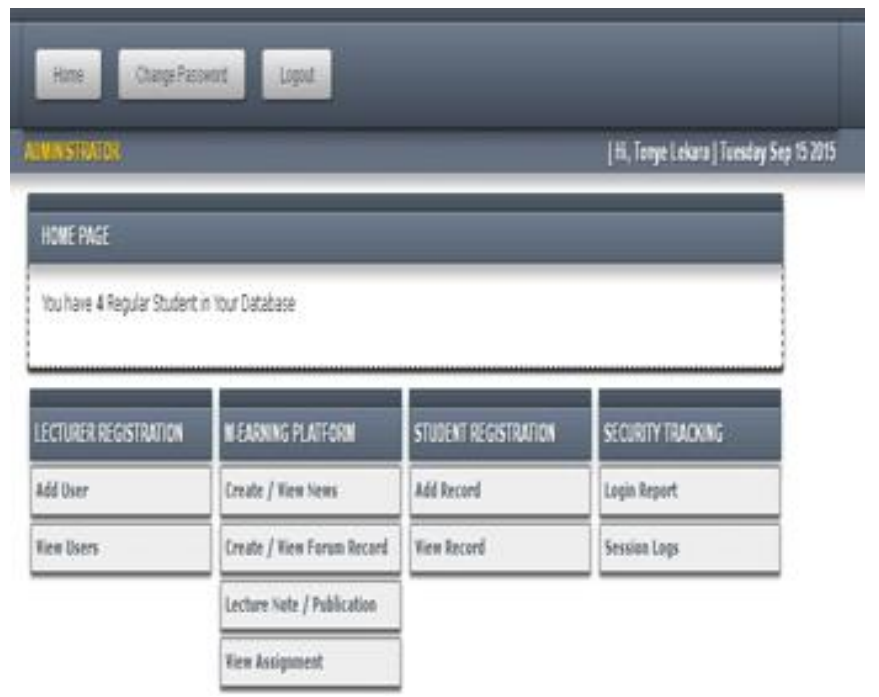

Figure 8: Admin Home Page Portal

The home page contains four major tabs. These include: Lecturer registration, M-Learning Portal, Student registration, and Security Tracking. From the Lecturer Registration tab, the Administrator can register new lecturers and also view the registered lecturers in the platform. The students "e registration involves more details about the students being registered. The necessary details about each intending student are filled out in this platform to create the students profile. For the M-Learning Portal tab, it is a link to the various interactive sessions such as creating news, creating forum sessions, upload lecture notes and publications, and also to view assignments. The Security Tracking tab keeps a record list of all users and their login time and session activities (whether active or inactive). It keeps track of users and their interactive sessions. 


\section{International Journal of Science and Research (IJSR) \\ ISSN (Online): 2319-7064 \\ Index Copernicus Value (2013): 6.14 | Impact Factor (2015): 6.391}

\subsection{Student Home Page}

The student logs into his account by entering the URL http://www.uniportmlearningportal.com in the web address bar which displays the above page (figure 9).

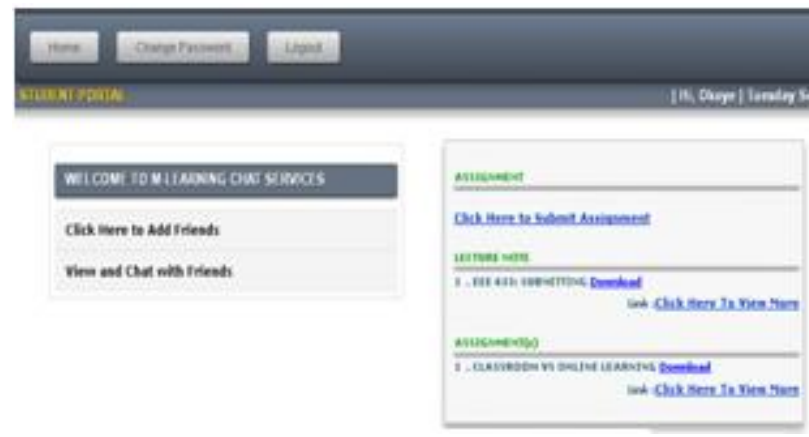

Figure 8: Student home page portal

(Figure 10) is the student registration tab, select ADD RECORD to open the registration portal. The required fields include: The students registration number, full name, Gender, date of birth, phone number, marital status, contact address, email address, department and date of registration. All fields are required to be completed to successfully create the students account.

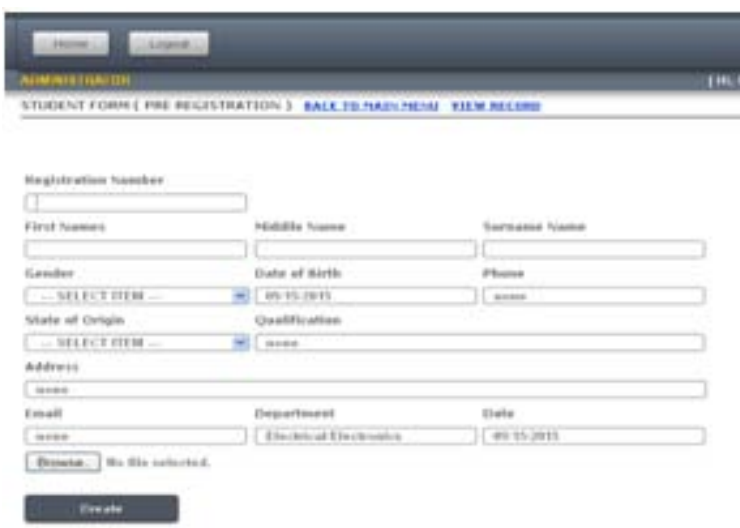

Figure 10: Student's Registration Platform

\subsection{Lecturers Registration Portal}

To create a new Lecturer account: Click on the drop down arrow and select human resource. Next, input the lecturers name, a username and then a password. Then click on save entry to save the information on the server's database. It is this username and password that the lecturer is required to supply each time he login to his profile. The (figure 11) illustrates the login environment where the authentication is done.

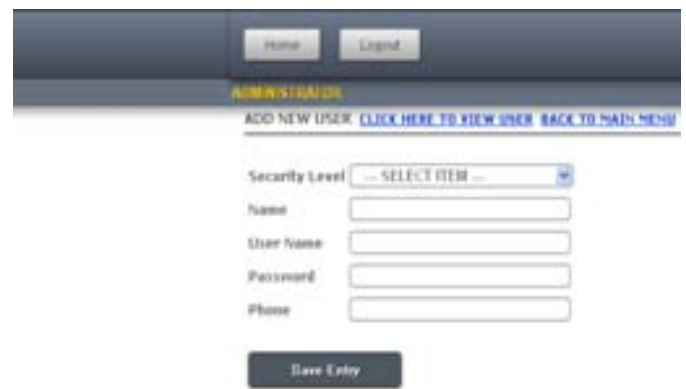

Figure 11: Lecturer's Registration Portal

\subsection{Online Group Discussion Forum}

This forum is an online discussion group platform (figure 12). It is an environment where participants can express their opinions or debate over a subject matter of interest and make necessary contributions to one another's questions. The forum is an interactive one by means of text. The subject of discussion is given a topic heading and a title.

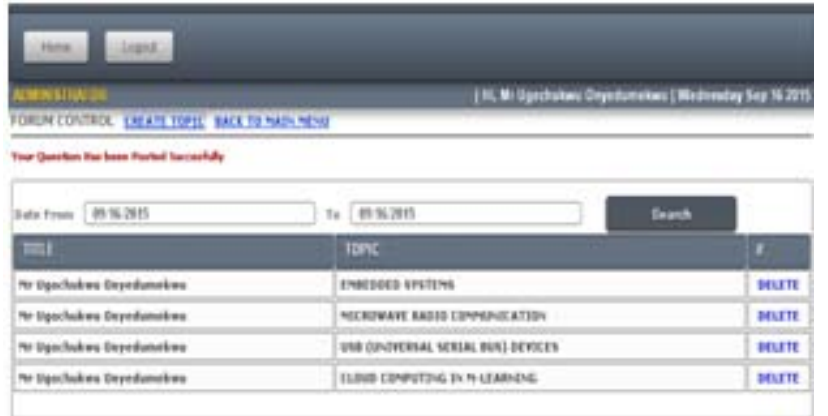

Figure 12: Online Group Discussion Forum

\subsection{Uploading Lecture Notes and Assignments Portal}

This is one of the fundamental aspects of the online learning platform. It is the vital aspect of the design that enables distance/online collaboration without the physical presence of the participants. This platform (figure 13) is designed to enable the lecturer upload lecture notes, tests and assignments by simply selecting the particular category of the uploaded document. When a student logs in into his or her account, the files can be easily downloaded by clicking the download button. As mentioned earlier, the attachments are not meant to be changeable or modified by the students. Thus, the attachment files are ensured to be in the PDF format which is a read-only document.

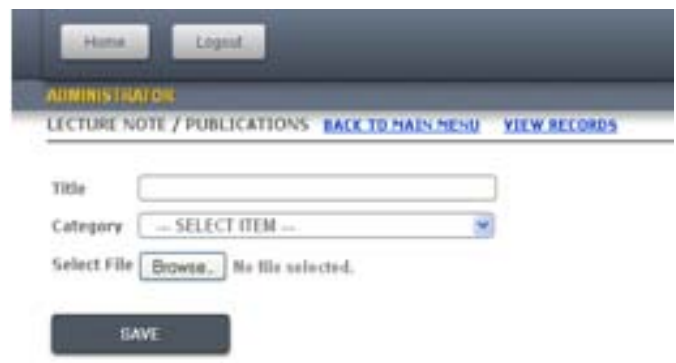

Figure 13: Lecture note/publication portal

\subsection{How to Open System application}

To open the application in offline mode, it can be launched from two different environments: The application has two login environments: Admin login and Student login. To login as a Student, type the address: $\mathrm{http}$ ://uniportmlearningportal.com in the browser. To login as an Admin, type the address: http://uniportmlearningportal.com/apps.

\section{Conclusion}

This research work has presented a new architecture of mobile learning system in a Cloud Computing environment 


\section{International Journal of Science and Research (IJSR) \\ ISSN (Online): 2319-7064}

Index Copernicus Value (2013): 6.14 | Impact Factor (2015): 6.391

enriched with mobile and wireless devices. An interactive mobile learning system in a cloud environment has provided a practical and cost-efficient solution. Mobile devices nowadays are widespread and provide great multimedia capabilities, which make the delivery of mobile learning a more realistic approach since it can provide just in time learning on the move. Where Mobile learning systems usually require many hardware and software resources, there are numerous educational institutions that cannot afford such investments, and cloud computing is the best solution for them. The main advantage of the cloud computing is that it has centralizing storage, memory, processing, bandwidth and also it optimizes the cost effectiveness for the implementation of the Hardware, software and License for all. Cloud computing and its concept in the field of education promote a new era of learning. Educational institutions are beginning to take advantage of existing applications hosted on a cloud that enable end users to perform tasks that have usually required site licensing, installation, and maintenance of individual software packages.

\section{References}

[1] S. B. Dash and H. Saini , "A Theoretical Aspect of Cloud Computing Service Models and its Security Issues": $A$ Paradigm: International Journal of Engineering Research and Applications. ISSN: 2248 9622, Vol. 4, Issue 6 (Version 1), June 2014, pp 248-254, 2014.

[2] Utpal Jyoti Bora and Majidul Ahmed, "E-Learning Using Cloud Computing": International Journal of Information Science and Modern Engineering, 2013.

[3] Keith Jeffery [ERCIM] and Burkhard Neidecker-Lutz , "The Future of Cloud Computing: Opportunities for European Cloud Computing beyond 2010”. European Commission: Information Society and Media.

[4] Abraham E. Eviwiekpaefe, "The Trend and Challenges of Cloud Computing: A literature Review”. International Letters of Social and Humanistic Sciences; Vol. 16, pp. 13-20, 2014.

[5] Peter Mell and Timothy Grance, "The NIST Definition of Cloud Computing": Recommendations of the National Institute of Standards and Technology; NIST Special Publication 800-145.2011.

[6] A Fernandez and D. Peralta, “An overview of E-Learning in Cloud Computing": Department of Computer Science, University of Jaen, Jaen, Spain, 2012.

[7] Haibo Yang and Mary Tate, "A Descriptive Literature Review and Classification of Cloud Computing Research", Communications of the Association for Information Systems: Article 2, Volume 13, 2012.

\section{Authors Profile}

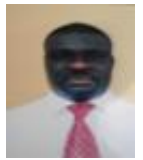

Georgewill Onengiye Moses got his B.Sc. in Computer Science at University of Nigeria Nsukka and first M.Sc in Applied Geophysics at Rivers State University of Science \& Technology Port Harcourt (RSUST).He also received his second (M.Eng) in Computer Engineering at Federal University of Technology Owerri (FUTO). Georgewill further had third master"s degree M.Sc in Computer Science at University of Port Harcourt (UNIPORT) and finally Ph.D in Computer Science in the same Institution. He is currently the Rector, Ken Saro Wiwa Polytechnic Rivers State, Nigeria. Georgewill has done over thirty publications. He is a member of Nigeria Computer Society (NCS), Association of Computer Machinery (ACM), Institute of Electrical and Electronic Engineering (IEEE), Computer Professionals (Registered Council) of Nigeria (CPN), International Research and Development Institute (IRDI). His research interest is in Artificial Intelligent (Fuzzy logic, Neural Network etc), Robotics, Computer Vision etc. He is happily married with children.

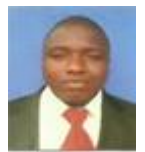

Ezeofor Chukwunazo Joseph received his B.Eng. in Computer Engineering at Enugu State University of Science and Technology (ESUT) and M.Eng in Communication Engineering at Federal University of Technology Owerri (FUTO). Ezeofor is currently studying Ph.D in Computer and Control Systems Engineering at Nnamdi Azikiwe University (NAU) Awka Nigeria. He has done over five publications and supervised more than fifteen students. He is a member Nigerian Society of Engineering (NSE) and registered member of Council of Regulation of Engineering in Nigeria (COREN). His research interest is on Real Time Embedded Systems, Control Systems, and Telecommunication. He is happily married with children. 